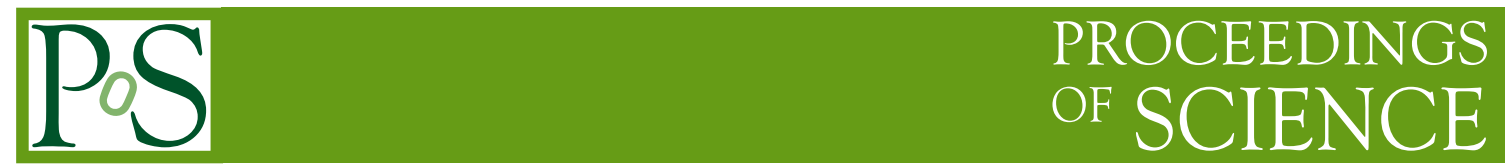

\title{
Higgs and Electroweak Measurements at the LHC
}

\author{
Michiel P. SANDERS ${ }^{* \dagger}$ \\ (Ludwig-Maximilians-Univeristät München $(D E)$ ) \\ E-mail: Michiel.Sanders@cern.ch
}

In this contribution, results on electroweak measurements and on Higgs boson searches at the Large Hadron Collider (LHC) are presented. The results are based on data collected by the ATLAS and CMS experiments at centre-of-mass energies of $7 \mathrm{TeV}$ and $8 \mathrm{TeV}$ with a total integrated luminosity of up to $25 \mathrm{fb}^{-1}$.

XXI International Workshop on Deep-Inelastic Scattering and Related Subject - DIS2013, 22-26 April 2013

Marseille, France

*Speaker.

On behalf of the ATLAS and CMS Collaborations. 


\section{Introduction}

The Large Hadron Collider (LHC) at CERN has provided the ATLAS [1] and CMS [2] experiments proton-proton collisions with an integrated luminosity of approximately $5 \mathrm{fb}^{-1}$ each at a centre-of-mass energy $(\sqrt{s})$ of $7 \mathrm{TeV}$ and approximately $20 \mathrm{fb}^{-1}$ each at $\sqrt{s}=8 \mathrm{TeV}$. In this contribution, the current status of electroweak measurements, Higgs boson searches and Higgs boson property measurements using these data is presented.

\section{Electroweak Measurements}

The theory of electroweak (EW) interactions is a quantum-field theory obeying local $S U(2) \times$ $U(1)$ gauge symmetries. This symmetry structure leads to couplings of fermions to the $\mathrm{W}$ and $\mathrm{Z}$ gauge bosons. In this way, production of single $\mathrm{W}$ or single $\mathrm{Z}$ bosons in proton-proton collisions at the LHC can be described. The theory also predicts couplings between three gauge bosons such as a $\gamma \mathrm{WW}$ and ZWW and between four gauge bosons such as $\gamma \gamma \mathrm{WW}$. These couplings can be probed in events where pairs of gauge bosons are produced (diboson production).

\subsection{Single W / Z Boson Production}

The CMS collaboration has measured the cross section for single $\mathrm{W}$ or $\mathrm{Z}$ boson production with a relatively small dataset of $\int \mathscr{L} \mathrm{d} t=18.7 \mathrm{pb}^{-1}$ collected at $\sqrt{s}=8 \mathrm{TeV}$ [3]. These data were taken at low instantaneous luminosity, to reduce the probability of multiple interactions per bunch-crossing (pile-up) and to allow for efficient low transverse momentum lepton triggers.

The selection of $\mathrm{Z}$ boson decays is based on identifying a pair of same-flavour electrons or muons with invariant mass close to the $\mathrm{Z}$ boson mass. For $\mathrm{W}$ boson decays, events with a single electron or muon are selected. The $\mathrm{Z}$ boson production cross section is then determined by counting the number of events compatible with a $\mathrm{Z}$ boson decay, whereas the $\mathrm{W}$ boson production cross section determination is based on a fit of a background and signal model to the distribution in data of the missing transverse energy (caused by the non-detected neutrino in the leptonic $\mathrm{W}$ boson decay). The event counts are then corrected for selection efficiencies and detector acceptance to inclusive cross sections, or to ratios of cross sections. An example is given in Fig. 1, which displays the $\mathrm{Z}$ boson production cross section versus the $\mathrm{W}$ boson production cross section. The uncertainty contours for the data indicate the correlations between systematic uncertainties, such as lepton identification uncertainties and uncertainties in the acceptance correction. The measured cross sections are in fair agreement with the predictions of the standard model using common choices for the parton distribution functions.

Properties such as the transverse momentum or the rapidity of the gauge bosons are the effect of the strong interaction, and are described in a different contribution to this conference.

\subsection{Diboson Production}

Observing and measuring the production of pairs of electroweak gauge bosons is not only another important test of the electroweak theory, but also gives constraints on possible anomalous gauge boson self-couplings. Moreover, diboson production is relevant for Higgs boson searches, 


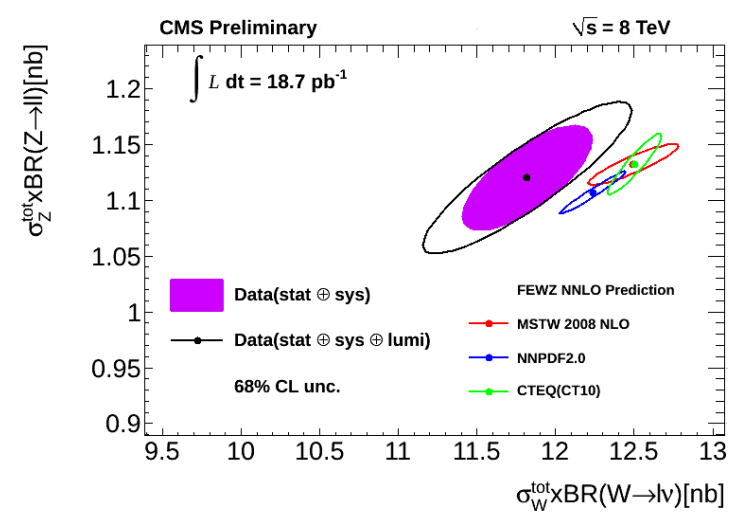

Figure 1: Cross sections of inclusive $\mathrm{Z}$ boson production and $\mathrm{W}$ boson production [3]. The black point indicates the measurements, whereas the coloured points and contours indicate the expectation from the standard model, for different choices of parton distribution function.

where it may appear as a significant source of background. Both the ATLAS and CMS collaborations have performed a full scan of possible diboson processes ( $\mathrm{W} \gamma, \mathrm{Z} \gamma, \mathrm{WW}, \mathrm{WZ}, \mathrm{ZZ}$ ) of which some are described in the following.

$\mathbf{W} \gamma$ and $\mathbf{Z} \gamma$ Production The ATLAS collaboration has used an integrated luminosity of $\int \mathscr{L} \mathrm{d} t=$ $4.7 \mathrm{fb}^{-1}$ at $\sqrt{s}=7 \mathrm{TeV}$ to measure inclusive and differential cross sections for $\mathrm{W} \gamma$ and $\mathrm{Z} \gamma$ production [4]. The event selection is based on identified leptons (electrons or muons) in the detector, significant missing transverse energy (for the $\mathrm{W} \gamma$ channel) and a photon. This photon has to be well separated from a lepton $(\Delta R(\ell, \gamma)>0.7)$ to reduce the contribution from events where the photon is radiated off a final state lepton. Non-signal backgrounds arise from, e.g., W/Z boson production with additional jets, where a jet is misidentified as a photon. As an example, in the $\mathrm{W} \gamma$ channel, 18313 events are found in data, with an expected background of approximately 7500 events of which $70 \%$ are from $\mathrm{W} / \mathrm{Z}+$ jets production.

Cross sections as function of photon transverse energy $\left(E_{\mathrm{T}}^{\gamma}\right)$ are shown in Fig. 2. For this, the experimental data are unfolded for detector resolution effects, and corrected for the detector acceptance up to a fiducial region that corresponds to the event selection criteria. For the exclusive case (number of jets equal to zero), there is rather good agreement between data and expectation (leading order generators scaled to the observed data, and MCFM at next-to-leading order [4]). For the inclusive case (any number of jets), the disagreement with the MCFM prediction can be attributed to missing higher order QCD contributions.

WW Production Production of a pair of W bosons was studied by the CMS collaboration with part of the dataset at $\sqrt{s}=8 \mathrm{TeV}\left(\int \mathscr{L} \mathrm{d} t=3.5 \mathrm{fb}^{-1}\right)$ [5]. The cross section measurement relies on the identification of two leptons (electron or muon) from leptonic $\mathrm{W}$ boson decays. To reduce background from $\mathrm{Z} / \gamma^{*} \rightarrow \ell^{+} \ell^{-}$production, large missing transverse energy is required, and events with two same-flavour leptons with an invariant mass close to the $\mathrm{Z}$ boson mass are rejected. Moreover, events with high transverse momentum jets are removed to reduce background from t⿱t production. In data, 1111 events passing these criteria are found, with an expected background of 275 events from dominantly t $\bar{t}$ and $\mathrm{W}+$ jets production. The inclusive $\mathrm{WW}$ production cross section is found 


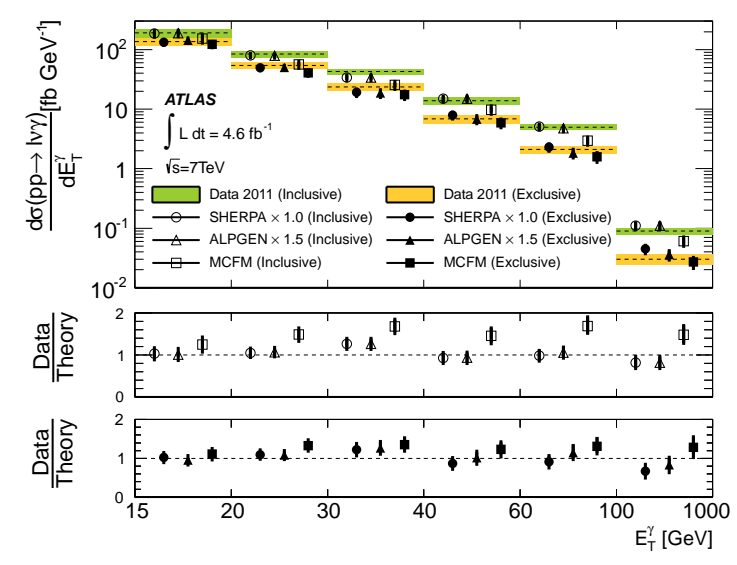

(a)

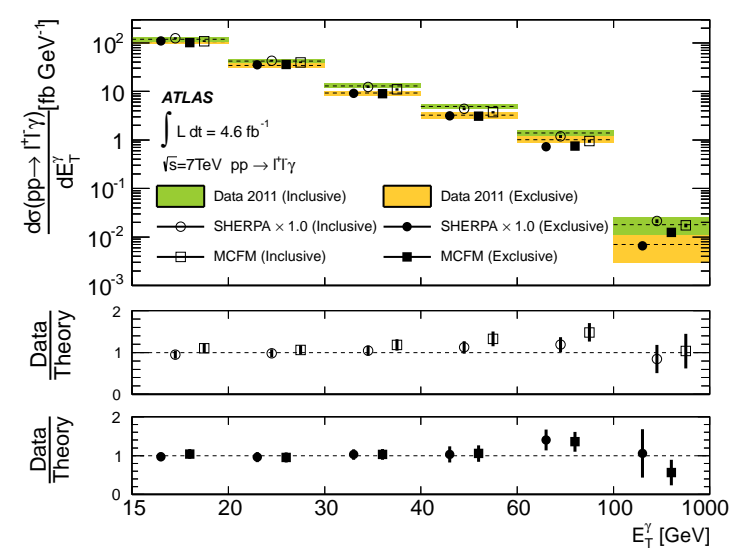

(b)

Figure 2: Fiducial cross sections as function of $E_{\mathrm{T}}^{\gamma}$, for the $\mathrm{W} \gamma$ channel (a) and $\mathrm{Z} \gamma$ channel (b), and ratios of data to theory prediction [4], for an exclusive selection (exactly zero jets in the final state) and inclusive selection (any number of jets).

to be $\sigma(\mathrm{pp} \rightarrow \mathrm{WW})=69.9 \pm 2.8$ (stat.) \pm 5.6 (syst.) \pm 3.1 (lum.) $\mathrm{pb}$. This is slightly higher than the expectation from the standard model of $\sigma(\mathrm{pp} \rightarrow \mathrm{WW})=57.3_{-1.6}^{+2.3} \mathrm{pb}$, calculated at next-to-leading order. Contributions from, e.g., Higgs boson production would increase the cross section by as much as $5 \%$.

The inclusive cross sections for WW production at $\sqrt{s}=7 \mathrm{TeV}$ measured by the CMS [6] and ATLAS [7] collaborations are slightly but not significantly larger than the standard model expectation. With the dataset at $\sqrt{s}=7 \mathrm{TeV}$, the ATLAS collaboration also measured the normalized fiducial (i.e., within the detector and event selection acceptance) cross section as function of the leading lepton transverse momentum, unfolded for detector resolution effects [7]. The result is shown in Fig. 3(a), and is in agreement with the standard model prediction.

WZ Production For WZ production, the experimental signature is events with three leptons (electrons, muons). The ATLAS collaboration has looked for this signature in data collected at $\sqrt{s}=8 \mathrm{TeV}\left(\int \mathscr{L} \mathrm{d} t=13 \mathrm{fb}^{-1}\right)[8]$. To identify the $\mathrm{Z}$ boson, two same-flavour leptons are required to have an invariant mass close to the $\mathrm{Z}$ boson mass; the $\mathrm{W}$ boson is identified by large missing transverse energy, and a large transverse mass of the third lepton and the missing transverse energy. Non-WZ background is caused by ZZ production or $\mathrm{Z}+$ jets production where a jet is misidentified as a lepton. Of the 1094 candidate events observed in data, 277 events are expected to be non$\mathrm{WZ}$ background. The inclusive $\mathrm{WZ}$ production cross section is then measured as $\sigma(\mathrm{pp} \rightarrow \mathrm{WZ})=$ $20.3_{-0.7}^{+0.8}$ (stat.) $)_{-1.1}^{+1.2}$ (syst. ${ }_{-0.6}^{+0.7}$ (lum.) pb, in excellent agreement with the standard model expectation of $\sigma(\mathrm{pp} \rightarrow \mathrm{WZ})=20.3 \pm 0.8 \mathrm{pb}$ (calculated at next-to-leading order).

Also at $\sqrt{s}=7 \mathrm{TeV}$, both the ATLAS and CMS collaboration measured cross sections for WZ production in agreement with the standard model expectation [9].

ZZ Production The production of a pair of $\mathrm{Z}$ bosons is the diboson production process with the smallest cross section. However, the experimental signature is very clean, if only decays of both $\mathrm{Z}$ bosons into leptons are considered. The ATLAS collaboration used the full dataset at $\sqrt{s}=8 \mathrm{TeV}$ 


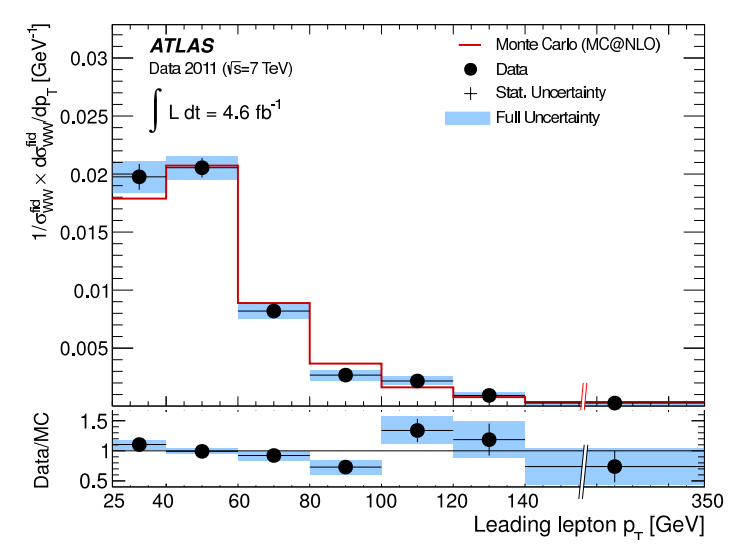

(a)

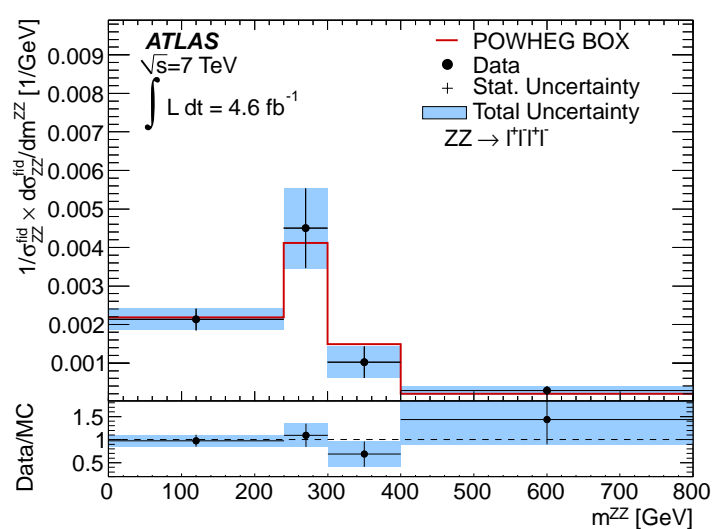

(b)

Figure 3: (a) Normalized fiducial cross section for WW production as function of the transverse momentum of the leading lepton [7], (b) normalized fiducial cross section for ZZ production as function of the $\mathrm{ZZ}$ invariant mass [12].

$\left(\int \mathscr{L} \mathrm{d} t=20.3 \mathrm{fb}^{-1}\right)$ to measure this cross section [10]. Events with four identified leptons are selected (electrons, muons), where pairs of same-flavour leptons are required to have an invariant mass close to the mass of the $\mathrm{Z}$ boson. Non-ZZ background originates from $\mathrm{Z}+$ jets and tt production where jets are misidentified as leptons, and is estimated directly from the data. Of the 305 events selected in the data, 20 are expected to be caused by background processes. The inclusive $\mathrm{ZZ}$ production cross section is then $\sigma(\mathrm{pp} \rightarrow \mathrm{ZZ})=7.1_{-0.4}^{+0.5}$ (stat.) \pm 0.3 (syst.) \pm 0.2 (lum.) pb; the standard model predicts $\sigma(\mathrm{pp} \rightarrow \mathrm{ZZ})=7.2_{-0.2}^{+0.3} \mathrm{pb}$ (at next-to-leading order). A similar measurement performed by the CMS collaboration at $\sqrt{s}=8 \mathrm{TeV}$ yields $\sigma(\mathrm{pp} \rightarrow \mathrm{ZZ})=8.4 \pm 1.0 \pm 0.7 \pm 0.4 \mathrm{pb}$ (standard model prediction $\sigma(\mathrm{pp} \rightarrow \mathrm{ZZ})=7.7 \pm 0.4 \mathrm{pb}$ ) [5]. The small difference in cross section between the two experiments is partially due to a different dilepton invariant mass window.

At the lower centre-of-mass energy $\sqrt{s}=7 \mathrm{TeV}$, the CMS [11] and ATLAS [12] collaborations found the cross section to also be in agreement with the standard model. In addition, the ATLAS collaboration measured unfolded differential cross sections for $\mathrm{ZZ}$ production at $\sqrt{s}=7 \mathrm{TeV}$. An example of that is given in Fig. 3(b) where the normalized fiducial cross section as function of the invariant mass of the $\mathrm{Z}$ boson pair is shown. The data are well described by the standard model (at next-to-leading order). It should be remarked that the statistical power of the data is still limited.

Limits on Anomalous Triple Gauge Boson Couplings The measurements of diboson production cross sections described before can be interpreted as measurements of the couplings between three gauge bosons (triple gauge coupling, TGC). This is typically done by setting limits on anomalous TGC's. The sensitivity is optimized by using kinematic properties of the observed events, such as the $\mathrm{Z}$ boson transverse momentum (in case of WZ production) or the transverse momentum of the leading lepton (in WW production). An example is given in Fig. 4 [13] for deviations from the standard model WWZ coupling. No deviations are found, and even with rather small datasets, the results from the ATLAS and CMS collaborations on WWZ and WW $\gamma$ couplings are reaching the world's leading sensitivity. For the ZZZ and ZZ $\gamma$ couplings, ATLAS and CMS have better sensitivity than previous experiments. 


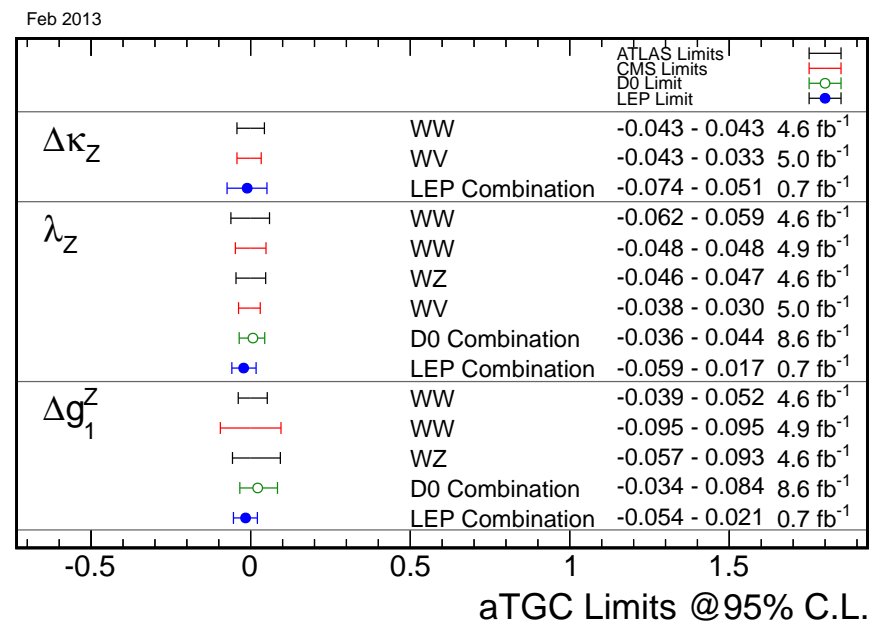

Figure 4: Limits on anomalous WWZ couplings [13].

\subsection{Forward-Backward Asymmetry}

The electroweak theory predicts an asymmetry in the momentum direction of leptons produced in the process $\mathrm{q} \overline{\mathrm{q}} \rightarrow \mathrm{Z} / \gamma^{*} \rightarrow \ell^{+} \ell^{-}$with respect to the direction of the incoming quark, in the restframe of the dilepton system. This forward-backward asymmetry has been measured by the ATLAS collaboration at $\sqrt{s}=7 \mathrm{TeV}\left(\int \mathscr{L} \mathrm{d} t=4.8 \mathrm{fb}^{-1}\right)$ [14], using dilepton pairs (electrons, muons). The unknown incoming quark direction is approximated by the longitudinal momentum direction of the dilepton system. This reduces the dilution in the asymmetry, defined as $A_{\mathrm{FB}}=\frac{\sigma_{\mathrm{F}}-\sigma_{\mathrm{B}}}{\sigma_{\mathrm{F}}+\sigma_{\mathrm{B}}}$, where $\sigma_{\mathrm{F}}\left(\sigma_{\mathrm{B}}\right)$ refers to the cross section for dilepton events with the positive (negative) decay angle. The dilution effect is further reduced by including events with an electron in the forward detector region (up to $\left|\eta_{\mathrm{e}}\right|=4.9$ ). The shape of the asymmetry is used to measure the weak mixing angle $\sin ^{2} \theta_{\mathrm{W}}$. It is found to be $\sin ^{2} \theta_{\mathrm{W}}^{\text {eff }}=0.2297 \pm 0.0004$ (stat.) \pm 0.0009 (syst.). This result is as precise as the most precise measurement at a hadron collider from $\mathrm{D} \emptyset$, and is in agreement with the world average.

\section{Higgs Boson Searches and Measurements}

To get a predictive theory of electroweak interactions with massive gauge bosons and fermions, the Higgs mechanism has been introduced. In its simplest form, an additional field, the Higgs field, breaks the electroweak symmetry and causes $\mathrm{W}$ and $\mathrm{Z}$ bosons to become massive. It also predicts the existence of a boson with spin-parity quantum numbers $J^{P}=0^{+}$, the Higgs boson. Fermion masses can be introduced by a direct coupling between the Higgs field and fermions.

To prove the validity of the Higgs mechanism, the Higgs boson has to be found and its properties measured. At the LHC, the Higgs boson is dominantly produced in the gluon-fusion process (through a top-quark loop). Sub-dominant is weak vector-boson fusion (VBF) process, and at an even smaller rates is the production of a Higgs boson in association with a $\mathrm{W}$ or $\mathrm{Z}$ boson or with a $\mathrm{t} \overline{\mathrm{t}}$ pair. The current LHC data allow for first measurements of its mass, spin-parity and its couplings to electroweak gauge bosons and fermions. In the following, a selection of Higgs boson searches and property measurements is given. Other results can be found in [15]. 


\subsection{Higgs Boson Search and Observation}

$\mathbf{H} \rightarrow \mathbf{Z Z}$ Decays of the Higgs boson into a pair of $\mathbf{Z}$ bosons which then decay to charged leptons give a very clean signal. The Higgs boson can be fully reconstructed from the four leptons, and background processes (dominantly non-resonant $\mathrm{ZZ}$ production and $\mathrm{Z}+$ jets production) are relatively rare. An example of the four-lepton invariant mass in this channel is given in Fig. 5, based on data from the CMS experiment $\left(\sqrt{s}=8 \mathrm{TeV}, \int \mathscr{L} \mathrm{d} t=19.6 \mathrm{fb}^{-1}\right)$ [16]. At a mass of $125 \mathrm{GeV}$, a clear peak is visible (expected mass resolution of $\sim 2 \mathrm{GeV}$ ), consistent with the decay of a Higgs boson. Combining the mass distribution with other kinematical variables, the significance of this excess is at the level of 6.7 standard deviations. Interpreted differently, a signal is observed with a strength $\mu$ relative to the standard model Higgs boson expectation of $\mu=\sigma / \sigma_{\mathrm{SM}}=0.91_{-0.24}^{+0.30}$ and a mass of $m_{\mathrm{H}}=125.8 \pm 0.5$ (stat.) \pm 0.2 (syst.) $\mathrm{GeV}$.

$\mathbf{H} \rightarrow \mathbf{W W}$ At a Higgs boson mass of $m_{\mathrm{H}}=125 \mathrm{GeV}$, the decay $\mathrm{H} \rightarrow \mathrm{WW}$ is the most dominant decay mode, after the $\mathrm{H} \rightarrow \mathrm{b} \bar{b}$ decay. A possible experimental signature is that of two leptons (electrons, muons) and large missing transverse energy, for the case that both $\mathrm{W}$ bosons decay leptonically. To increase the search sensitivity, the events can be classified according to the number of jets. The background from tit production can be reduced by removing events with jets with a b-hadron signature. Remaining backgrounds are then non-resonant WW production, $t \bar{t}$ production and Drell-Yan dilepton production. Due do the non-observable neutrinos, the mass of the Higgs boson cannot be reconstructed. Instead, the transverse mass (of the two leptons and the missing transverse energy) can be used, as shown in Fig. 6 for the ATLAS analysis using $\int \mathscr{L} \mathrm{d} t=20.7 \mathrm{fb}^{-1}$ at $\sqrt{s}=8 \mathrm{TeV}$ [17]. A clear excess can be seen, consistent with standard model Higgs boson production. For a mass of $m_{\mathrm{H}}=125 \mathrm{GeV}$, an excess over background of $3.8 \sigma$ is observed (including data at $\sqrt{s}=7 \mathrm{TeV}$ ), and a signal strength of $\mu=1.01 \pm 0.31$ is measured. This ATLAS analysis by itself also excludes Higgs boson masses above $m_{\mathrm{H}}=133 \mathrm{GeV}$, at $95 \%$ confidence level (CL).

The CMS collaboration also looked for $\mathrm{H} \rightarrow \mathrm{WW}$ decays in events with a third identified lepton [18]. This search is directly sensitive to associated WH production. This production and decay mode is only dependent on the HWW coupling. After rejecting events with additional jets to remove $t \bar{t}$ background, the dominant background is $\mathrm{WZ}$ and $\mathrm{Z}+$ jets production. With the present data, the search can exclude 3.3 times the expected standard model Higgs boson cross section, at $m_{\mathrm{H}}=125 \mathrm{GeV}$ and $95 \% \mathrm{CL}$.

$\mathbf{H} \rightarrow \gamma \gamma$ The $\mathrm{H} \rightarrow \gamma \gamma$ decay has a very small branching ratio, but experimentally it is easy to observe as a narrow peak in the diphoton invariant mass distribution (with a typical experimental mass resolution of less than $2 \mathrm{GeV}$ ) on top of a smooth falling background. By splitting the events in various jet multiplicity bins and in photon reconstruction quality categories, the sensitivity is optimized. The CMS collaboration used photon identification algorithms based on multi-variate techniques to get an even better sensitivity than with cut-based algorithms [19]. Using the full dataset $\left(\int \mathscr{L} \mathrm{d} t=5.1 \mathrm{fb}^{-1}\right.$ at $\sqrt{s}=7 \mathrm{TeV}, \int \mathscr{L} \mathrm{d} t=19.6 \mathrm{fb}^{-1}$ at $\left.\sqrt{s}=8 \mathrm{TeV}\right)$, the CMS analysis gives a signal strength of $\mu=0.78_{-0.26}^{+0.28}$ (in agreement with the standard model value $\mu=1$ ) and a Higgs boson mass of $m_{\mathrm{H}}=125.4 \pm 0.5$ (stat.) \pm 0.6 (syst.) $\mathrm{GeV}$. A cut-based analysis on the same dataset gives a larger observed signal strength, which is also compatible with the standard model and with the multi-variate analysis. 


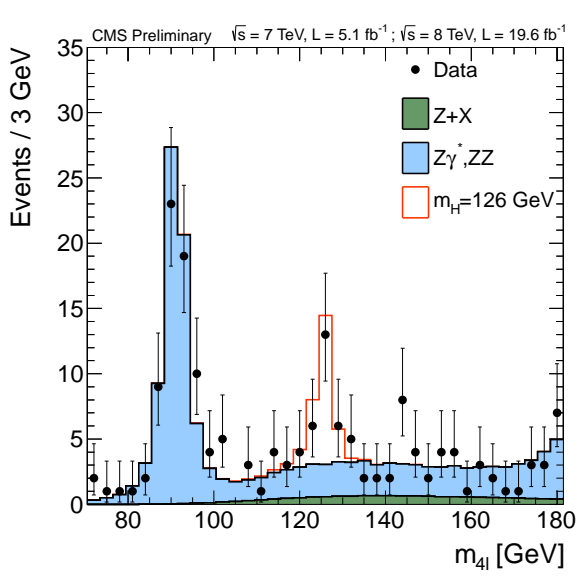

Figure 5: Invariant mass of four leptons (electrons, muons) in the $\mathrm{H} \rightarrow \mathrm{ZZ}$ search [16].

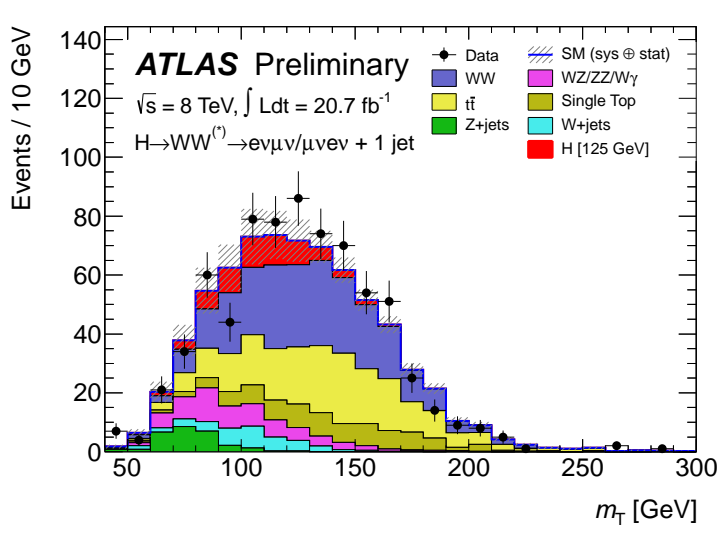

Figure 6: Transverse mass of leptons and missing transverse energy in the $\mathrm{H} \rightarrow \mathrm{WW}$ search [17].

$\mathbf{H} \rightarrow \tau \tau / \mu \mu$ Observing Higgs boson decays to a pair of tau leptons, muons or bottom quarks would prove directly the coupling of the Higgs boson to fermions. The most sensitive fermionic decay mode is that to tau leptons. The tau leptons themselves decay further to lighter leptons or hadrons. In an analysis by the CMS collaboration, using data taken at $\sqrt{s}=7 \mathrm{TeV}$ and $\sqrt{s}=8 \mathrm{TeV}$ [20], five final states were studied separately: $\tau \tau \rightarrow \mu \tau_{\mathrm{h}} / \mathrm{e} \tau_{\mathrm{h}} / \mathrm{e} \mu / \tau_{\mathrm{h}} \tau_{\mathrm{h}} / \mu \mu$, where $\tau_{\mathrm{h}}$ indicates a hadronic tau lepton decay. The dominant background from $\mathrm{Z} \rightarrow \tau \tau$ events is estimated using an "embedded" sample, which is a $\mathrm{Z} \rightarrow \mu \mu$ event sample from data in which the muons are replaced by simulated tau lepton decays. For events with two identified high transverse energy jets, specific VBF cuts are applied to enhance the sensitivity to this production mode. Fig. 7 shows the di-tau invariant mass distribution. A $2.9 \sigma$ excess over the expected background is visible, consistent with Higgs boson production. The measured signal strength at a mass of $m_{\mathrm{H}}=125 \mathrm{GeV}$ is $\mu=1.1 \pm 0.4$, whereas the measured Higgs boson mass is $m_{\mathrm{H}}=120_{-7}^{+9} \mathrm{GeV}$.

The branching ratio for the decay of a Higgs boson to a muon pair is rather small, but still a factor of two larger than the decay $\mathrm{H} \rightarrow \mathrm{ZZ} \rightarrow 4 \mathrm{e} / 4 \mu / 2 \mathrm{e} 2 \mu$ (at $m_{\mathrm{H}}=125 \mathrm{GeV}$ ). Observing these $\mathrm{H} \rightarrow \mu \mu$ decays is still challenging because of the large Drell-Yan dimuon production background. The ATLAS collaboration used data collected at $\sqrt{s}=8 \mathrm{TeV}\left(\int \mathscr{L} \mathrm{d} t=20.7 \mathrm{fb}^{-1}\right)$ [21] to search for a narrow $\mathrm{H} \rightarrow \mu \mu$ peak in the invariant mass distribution (expected mass resolution $\sim 2.5 \mathrm{GeV}$ ), as shown in Fig. 8. With these data, cross sections for standard model Higgs boson production larger than 9.8 times the standard model expectation can be excluded (at $m_{\mathrm{H}}=125 \mathrm{GeV}$ and $95 \% \mathrm{CL}$ ).

\subsection{Higgs Boson Properties}

Mass As described in the previous paragraphs, the $\mathrm{H} \rightarrow \mathrm{ZZ} \rightarrow 4 \ell$ and $\mathrm{H} \rightarrow \gamma \gamma$ channels have excellent mass resolution and show significant evidence of Higgs boson production at a mass of approximately $125 \mathrm{GeV}$. These channels are therefore combined by both the ATLAS and CMS collaborations using all available data to give more precise measurements of the observed resonance. In the ATLAS combination [22], a mass of $m_{\mathrm{H}}=125.5 \pm 0.2$ (stat. $)_{-0.6}^{+0.5}$ (syst.) $\mathrm{GeV}$ is measured. The CMS mass measurement [23] yields $m_{\mathrm{H}}=125.7 \pm 0.3$ (stat.) \pm 0.3 (syst.) $\mathrm{GeV}$. The ATLAS 


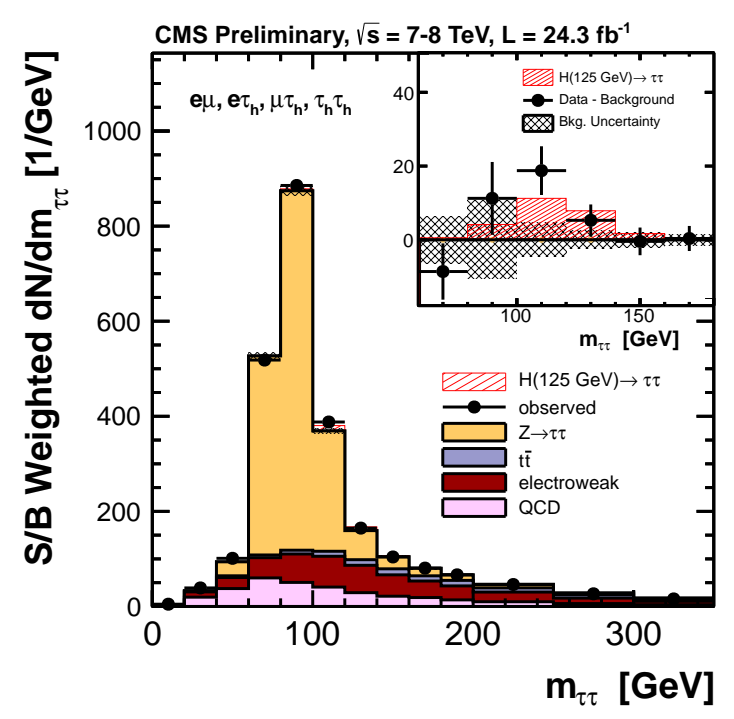

Figure 7: Di-tau invariant mass in the $\mathrm{H} \rightarrow \tau \tau$ search [20].

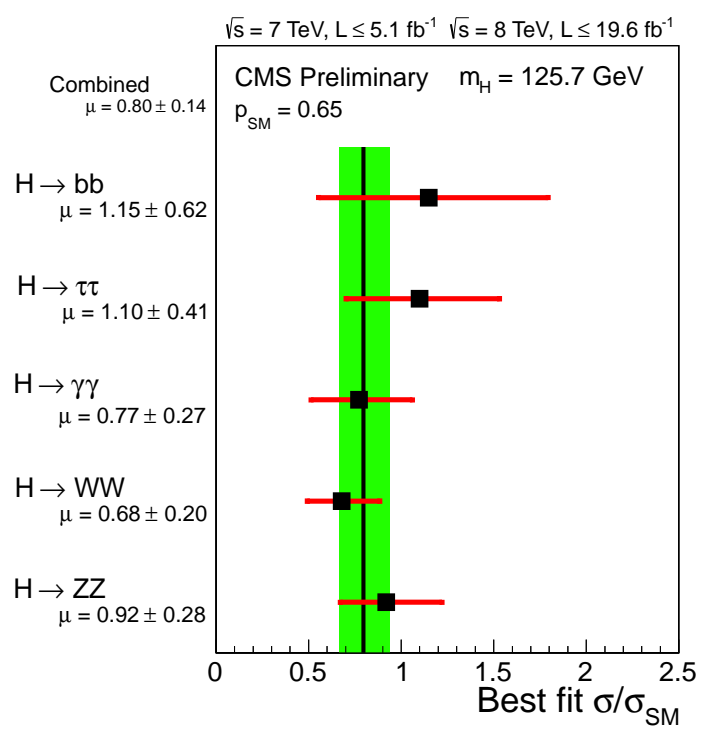

(a)

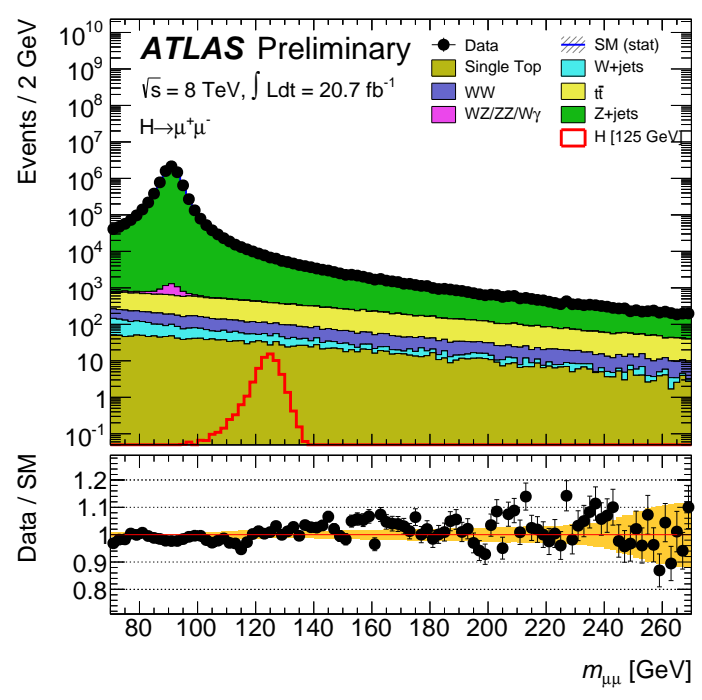

Figure 8: Di-muon invariant mass in the $\mathrm{H} \rightarrow$ $\mu \mu$ search [21].

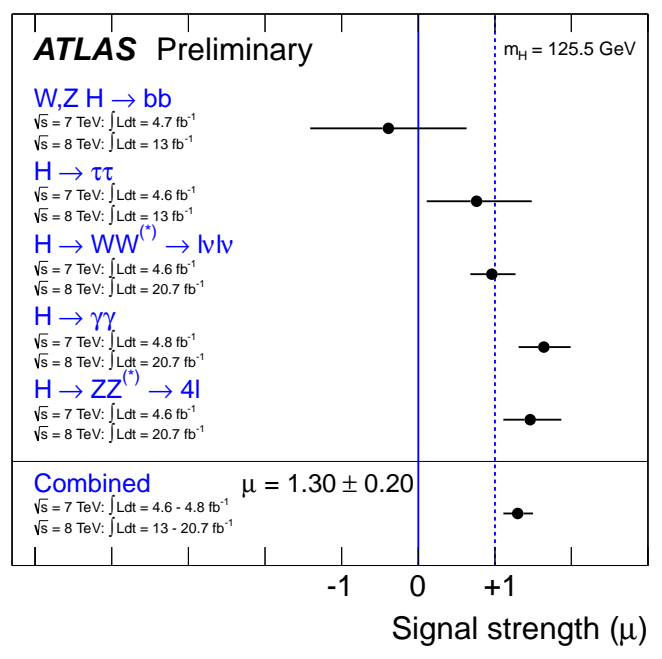

(b)

Figure 9: Higgs boson signal strength measurements from (a) the CMS collaboration [23] and from (b) the ATLAS collaboration [24].

measurements give a mass difference between the two decay channels of $\Delta m_{\mathrm{H}}=m_{\gamma \gamma}-m_{4 \ell}=$ $2.3_{-0.7}^{+0.6}$ (stat.) \pm 0.6 (syst.) $\mathrm{GeV}$, corresponding to a $2.5 \sigma$ deviation from the expected value of zero.

Signal Strength A summary of the observed signal strength $\mu=\sigma / \sigma_{\mathrm{SM}}$ for Higgs boson production in the various decay channels is given in Fig. 9(a) for the CMS measurements [23] and in Fig. 9(b) for the ATLAS measurements [24]. Combining all decay channels, the CMS collaboration finds $\mu=0.80 \pm 0.14$ and the ATLAS collaboration finds $\mu=1.30 \pm 0.20$. Within the uncertainties, all measurements are in agreement with the standard model expectation of $\mu=1$. 


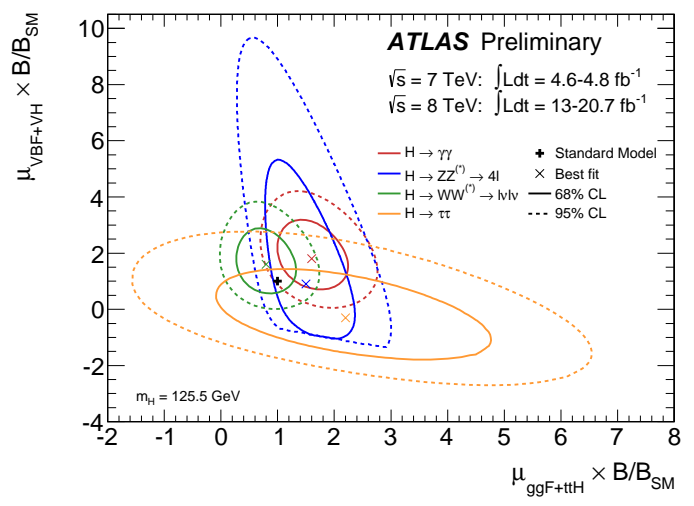

(a)

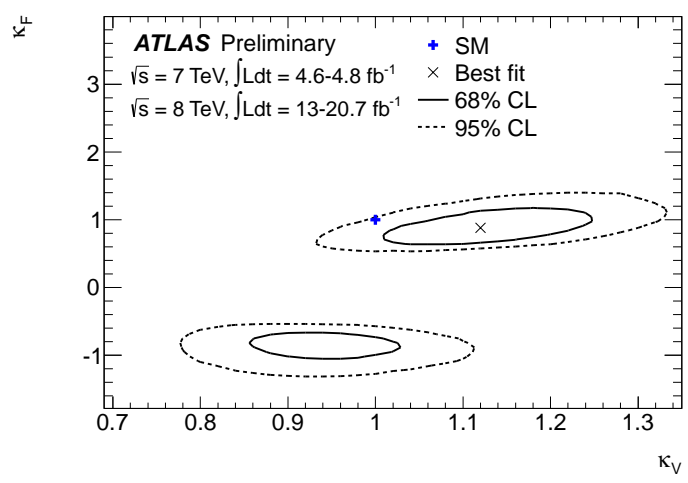

(b)

Figure 10: (a) Measured signal strength for Higgs boson production in various production and decay modes, (b) measured Higgs boson coupling scale factors [24].

Spin and Parity To test whether the observed Higgs boson-like excess has the spin and parity expected for a standard model Higgs boson $\left(J^{P}=0^{+}\right)$, the data can be compared to models with various spin-parity assumptions. The CMS collaboration used the $\mathrm{H} \rightarrow \mathrm{ZZ}$ data to fully reconstruct the decay angles [16]. From the various spin-parity combinations tested, the standard model value $J^{P}=0^{+}$is strongly favoured. The ATLAS collaboration used multi-variate techniques to test the $J^{P}=0^{+}$assignment against a benchmark $J^{P}=2^{+}$model (commonly labeled as the $2_{m}^{+}$model), using a subset of $\mathrm{H} \rightarrow \mathrm{WW}$ candidate events [25]. The $2_{m}^{+}$model is excluded at more than $95 \% \mathrm{CL}$, independent of the fraction of $2_{m}^{+}$production from gluon-fusion or from $\mathrm{q} \overline{\mathrm{q}}$ annihilation. Similarly, the ATLAS analysis of $\mathrm{H} \rightarrow \gamma \gamma$ events prefers the $J^{P}=0^{+}$assignment over $2_{m}^{+}$[26]. The ATLAS collaboration also combined tests of the $J^{P}=0^{+}$versus $2_{m}^{+}$hypotheses in various decay channels [27]. From this combination, the $2_{m}^{+}$state is excluded at more than $99.9 \% \mathrm{CL}$.

Couplings The production modes of the Higgs boson depend either on bosonic couplings (associated production, VBF) or fermionic couplings (gluon-fusion). The different search channels are sensitive to both, as shown in Fig. 10(a), based on data from the ATLAS collaboration [24]. These channels can be combined into a measurement on the ratio of bosonic production and fermionic production $\mu_{\mathrm{VBF}, \mathrm{VH}} / \mu_{\mathrm{gg} \rightarrow \mathrm{H}, \mathrm{tt} \mathrm{H}}=1.2_{-0.5}^{+0.7}$, constituting a more than $3 \sigma$ evidence for VBF production of Higgs bosons. The data can also be used to determine scale factors $\kappa$ for the coupling of the Higgs boson to electroweak bosons $\kappa_{\mathrm{V}}$ or to fermions $\kappa_{\mathrm{F}}$. Given the current data, these couplings are consistent with the expected value of one (Fig. 10(b)).

\section{Summary}

The collision data provided by the LHC to the ATLAS and CMS experiments so far have allowed for many tests of the electroweak theory. The measurements presented here are all consistent with the theory, and are approaching a precision comparable to previous experiments. In the searches for the Higgs boson, the observation of a signal around $m_{\mathrm{H}}=125.5 \mathrm{GeV}$ has been established more firmly than before. The properties of the observed state are compatible with that of a standard model Higgs boson, within the still rather large uncertainties. 


\section{References}

[1] ATLAS Collaboration, JINST 3 (2008) S08003.

[2] CMS Collaboration, JINST 3 (2008) S08004.

[3] CMS Collaboration, CMS-PAS-SMP-12-011, http://cds.cern.ch/record/1460098.

[4] ATLAS Collaboration, Phys. Rev. D 87, 112003 (2013).

[5] CMS Collaboration, Phys. Lett. B 721 (2013) 190.

[6] CMS Collaboration, arXiv:1306.1126 [hep-ex], CERN-PH-EP-2013-075.

[7] ATLAS Collaboration, Phys. Rev. D 87, 112001 (2013).

[8] ATLAS Collaboration, ATLAS-CONF-2013-021, http://cds.cern.ch/record/1525557.

[9] ATLAS Collaboration, Eur. Phys. J. C 72 (2013) 2173;

CMS Collaboration, CMS-PAS-EWK-11-010, http://cds.cern.ch/record/1370067.

[10] ATLAS Collaboration, ATLAS-CONF-2013-020, http://cds.cern.ch/record/1525555.

[11] CMS Collaboration, JHEP 01 (2013) 063.

[12] ATLAS Collaboration, JHEP 03 (2013) 128.

[13] http://twiki.cern.ch/twiki/bin/view/CMSPublic/PhysicsResultsSMPaTGC, 1 March 2013.

[14] ATLAS Collaboration, ATLAS-CONF-2013-043, http://cds.cern.ch/record/1544035.

[15] ATLAS Collaboration, https://twiki.cern.ch/twiki/bin/view/AtlasPublic; CMS Collaboration, https://twiki.cern.ch/twiki/bin/view/CMSPublic/PhysicsResults.

[16] CMS Collaboration, CMS-PAS-HIG-13-002, http://cds.cern.ch/record/1523767.

[17] ATLAS Collaboration, ATLAS-CONF-2013-030, http://cds.cern.ch/record/1527126.

[18] CMS Collaboration, CMS-PAS-HIG-13-009, http://cds.cern.ch/record/1523681.

[19] CMS Collaboration, CMS-PAS-HIG-13-001, http://cds.cern.ch/record/1530524.

[20] CMS Collaboration, CMS-PAS-HIG-13-004, http://cds.cern.ch/record/1528271.

[21] ATLAS Collaboration, ATLAS-CONF-2013-010, http://cds.cern.ch/record/1523695.

[22] ATLAS Collaboration, ATLAS-CONF-2013-014, http://cds.cern.ch/record/1523727.

[23] CMS Collaboration, CMS-PAS-HIG-13-005, http://cds.cern.ch/record/1542387.

[24] ATLAS Collaboration, ATLAS-CONF-2013-034, http://cds.cern.ch/record/1528170.

[25] ATLAS Collaboration, ATLAS-CONF-2013-031, http://cds.cern.ch/record/1527127.

[26] ATLAS Collaboration, ATLAS-CONF-2013-029, http://cds.cern.ch/record/1527124.

[27] ATLAS Collaboration, ATLAS-CONF-2013-040, http://cds.cern.ch/record/1542341. 